\title{
Mechanism and Biological Explanation*
}

\author{
William Bechtel ${ }^{\dagger+}$
}

This article argues that the basic account of mechanism and mechanistic explanation, involving sequential execution of qualitatively characterized operations, is itself insufficient to explain biological phenomena such as the capacity of living organisms to maintain themselves as systems distinct from their environment. This capacity depends on cyclic organization, including positive and negative feedback loops, which can generate complex dynamics. Understanding cyclically organized mechanisms with complex dynamics requires coordinating research directed at decomposing mechanisms into parts (entities) and operations (activities) with research using computational models to recompose mechanisms and determine their dynamic behavior. This coordinated endeavor yields dynamic mechanistic explanations.

1. Introduction. Within biology (and the life sciences more generally), there is a long tradition of explaining a phenomenon by describing the mechanism responsible for it. A perusal of biology journals and textbooks yields many mentions of mechanisms but few of laws. Philosophers of

*Received October 2010; revised January 2011.

$\dagger$ To contact the author, please write to: Department of Philosophy, University of California, San Diego, 9500 Gilman Drive, La Jolla, CA 92093-0119; e-mail: bill@mechanism .ucsd.edu.

$\$$ I thank Adele Abrahamsen, Colin Allen, Carl Craver, Lindley Darden, William Wimsatt; audiences at Duke University, Indiana University, University of the Basque Country, University of California, Irvine, University of Chicago, University of Groningen; and referees for this journal for many helpful comments and suggestions. This article's title is not original; it previously appeared as the title of a 1972 article in Philosophy of Science by Francisco Varela and Humberto Maturana. They noted the long prominence of mechanistic language in biology (e.g., in the vitalism/mechanism controversy), but their ultimate goal, quite different from that of this article, was to defend the position that came to be known as functionalism in philosophy of mind, according to which the proper characterization of a mechanism was abstract, not tied to particular realizations of components. In publications that began appearing shortly thereafter, however, they began to develop their conception of autopoiesis and an understanding of mechanism that is much closer to the position advanced in this article.

Philosophy of Science, 78 (October 2011) pp. 533-557. 0031-8248/2011/7804-0001\$10.00

Copyright 2011 by the Philosophy of Science Association. All rights reserved. 
science in the twentieth century exhibited the reverse asymmetry. A focus on certain domains of physics led them to construct the deductive-nomological (DN) framework (Hempel 1965), which emphasized laws and ignored mechanistic accounts. In the last 2 decades, though, several philosophers of science focusing on biology have offered analyses of mechanistic explanations as advanced by biologists. Initially, they converged on what I term the basic mechanistic account, ${ }^{1}$ in which a mechanism is construed as generating a phenomenon (e.g., protein synthesis) through a start-to-finish sequence of qualitatively characterized operations performed by component parts.

Biological explanations that adhere to the basic mechanistic schema have often been criticized as inadequate to explain biological phenomena. This perceived shortcoming was a major source of vitalist opposition to mechanistic biology throughout the eighteenth and nineteenth centuries. Although the vitalists' rejection of mechanism, per se, was unjustified, their objections to particular mechanistic explanations were well founded, as was their contention that the overall conception of mechanism employed was inadequate. This shortcoming has been inherited by the basic account of mechanism sketched above: by treating mechanisms as paradigmatically involving qualitatively characterized operations executed sequentially, it fails to accommodate key features of biological phenomena. This is not to deny that the basic account of mechanistic explanation represents a significant advance beyond earlier philosophical approaches to explanation and provides important insights into the explanatory pursuits of biologists. Rather, it points to the fact that while these accounts note the importance of organization, they do not address the forms of nonsequential organization characteristic of living systems and the challenges biologists confront in understanding the effects of such organization.

One way to appreciate the shortcomings of the basic account of mechanism is to recognize that it better fits Jacques de Vaucanson's (1709-82) "moving anatomy" than it does living organisms. Vaucanson, an engineer, developed exquisitely realistic mechanical models of animals. Perhaps best known was his mechanical duck, which could stand and sit, move its wings, drink water, eat and digest fish, and excrete the remnants. The responsible mechanisms comprised more than a thousand moving parts, which were concealed inside the duck and the base on which it stood, and relied on a sophisticated system of weights for energy (Riskin 2003).

Vaucanson's duck superficially exhibited many behaviors of real ducks. It was far from being alive, though, and the differences are as revealing

1. I will occasionally refer to the mechanisms envisaged by the basic account as basic mechanisms. 
as the behaviors it simulated. Although it ate, for example, that was not how it secured the energy needed to maintain itself as a functioning duck. Energy was provided by Vaucanson and his assistants, who set the weights that powered the actions of the duck. One of the features of living organisms that a few theorists, in both biology and philosophy, have begun to emphasize is their autonomy - their ability to maintain themselves as systems distinct from their environment by directing the flow of matter and energy so as to build and repair themselves. Vaucanson's duck lacked autonomy.

In the following section, I provide a brief introduction to the basic account of mechanism and mechanistic explanation, emphasizing both its distinctive contributions to philosophy of science and how it implicitly has figured in the remarkable advance in biological knowledge across the nineteenth and twentieth centuries. In section 3, I turn to the autonomy of biological organisms, a feature that gains greater prominence as investigators attempt to recompose and situate biological mechanisms in their environment. I address the particular kinds of cyclic organization by which biological organisms maintain their autonomy in sections 4 and 5 , and in section 6 I consider how cyclic organization figures in generating the endogenous activity that is characteristic of autonomous systems. In section 7, I turn back to mechanism and discuss how mechanistic accounts must forego a sequential perspective and be supplemented by computational modeling and dynamic analysis in order to adequately explain these characteristics of living organisms.

2. The Basic Mechanistic Account. Descartes is famous for promoting mechanistic explanation in opposition to the dominant Aristotelian approach that emphasized teleological explanation. He states, "I have described this earth and indeed the whole universe as if it were a machine: I have considered only the various shapes and movements of its parts" (Descartes Principia IV 188). Descartes' conception of a machine was one in which the shapes and motions of its hypothesized components (corpuscles) were invoked to explain its behavior-he allowed neither a vacuum nor action at a distance. Over the ensuing century, Boyle relaxed the prohibition against a vacuum, and Newton proposed to account for action at a distance in terms of laws involving forces. ${ }^{2}$ As Machamer, Darden, and Craver (2000) demonstrated, these restrictions have been further relaxed as some research fields treat as basic such activities as forming or breaking a hydrogen bond or conducting an electrical impulse. But the core idea-explaining natural phenomena by identifying the re-

2. There are conflicting interpretations of Newton as either expanding the mechanist perspective or repudiating it in favor of a nomological approach (see Boas 1952). 
sponsible mechanism and explaining its functioning in terms of its parts and the operations they perform - was adopted by many scientists, especially in the emerging biological sciences.

It is essentially this extended Cartesian conception of a mechanism that has been articulated by the new mechanistic philosophers of science. Wimsatt (1976) was one of the first philosophers to observe that biologists typically appeal to mechanisms, not theories or laws, in offering explanations. ${ }^{3}$ Building on Wimsatt's insight as well as those of Simon (1962) and Kauffman (1971), Bechtel and Richardson (1993/2010) emphasized the processes by which scientists decompose mechanisms structurally into their parts and functionally into their operations. Emphasizing the same distinction between parts and operations (which they call entities and activities), Machamer et al. (2000, 3) proposed their widely cited characterization of mechanisms: "Mechanisms are entities and activities organized such that they are productive of regular changes from start or set-up to finish or termination conditions." 4

The last phrase of Machamer, Darden, and Craver's characterization"from start or set-up to finish or termination conditions"-imposes a sequential ordering on the activities of the mechanism. The assumption of sequential order reflects the practices of many scientists, who attempt to envisage sequentially the qualitative changes occurring in the mechanisms they investigate. More fundamentally, this reflects the sequential nature of human mental processes. We perceive successive states of the world, and in imagination we redeploy perceptual processes (Kosslyn 1994) and so imagine changes sequentially. In explicit linguistic reasoning, we represent transformations in the world sequentially. Further, we naturally assume that such sequences of transformations will be repeated in qualitatively the same way in the future, unless changed conditions are explicitly noted. Assuming repeatable sequential ordering of qualitatively characterized operations is not only natural, it often provides a valuable first approach to explaining a biological process. Only with an initial sequential account in place have scientists been successful in recognizing and taking into account the departures from sequential ordering on which I focus in later sections of this article.

Accordingly, when seeking to understand how proteins are synthesized, scientists describe sequential operations of the RNA polymerase initiating

3. He contended: "At least in biology, most scientists see their work as explaining types of phenomena by discovering mechanisms, rather than explaining theories by deriving them from or reducing them to other theories, and this is seen by them as reduction, or as integrally tied to it" (Wimsatt 1976, 671).

4. For related characterizations of mechanism, see Glennan $(1996,2002)$ and Thagard (2003, 2006). 
the transcription of DNA into mRNA, the transport of mRNA (as well as tRNA and rRNA) into the cytoplasm, the operation of tRNA in pairing amino acids with codons on the mRNA, and finally the folding of the protein. Darden and Craver (2002) have shown how discovery of this mechanism resulted, in part, from reasoning forward from the DNA and backward from the sequence of amino acids to fill in the intermediate operations in an initial sketch. An important feature of this example, and of many others that have been considered by the new mechanistic philosophers of science, is that each of the operations in such a sequence is characterized qualitatively (e.g., "transcription of DNA into mRNA" with no quantitative specification of the possibly varying rate). If possible, the parts involved in each operation will be identified as well (e.g., the particular gene, mRNA, and RNA polymerase), including specification of the chemical structure of each. A basic mechanistic explanation combines these parts and operations, qualitatively specifying the spatial organization of the parts and the temporal sequence of operations that are performed in succession until the termination conditions are satisfied, in this way producing the phenomenon of interest.

The basic account of mechanistic explanation differs in many ways from the more traditional DN model of explanation, in which explanation is provided by laws and derivations from laws. It provides a distinct perspective on issues that have been central to philosophical discussions over the past century. First, the crucial component of a mechanistic account is not the formulation of the relevant law but the determination of the parts of the mechanism, the operations they perform, and how they are organized. Laws may be invoked to characterize the overall functioning of the mechanism or some of its operations, but it is the discovery that particular operations are being performed that is required to specify the mechanism. Second, although these parts, operations, and their organization can be described linguistically, it is often more productive to represent them in diagrams, with text serving as commentary to guide interpretation of the diagrams. Third, demonstration that the mechanism could produce the phenomenon does not rely on logical derivations but rather on mental simulations of the mechanism in operation.

In connection with these first three points, it is important to note that proposed accounts of mechanisms are often incomplete, and known to be such, even while they are being actively employed. Machamer et al. (2000) distinguish mechanism sketches (accounts with known gaps between operations) and mechanism schemas (accounts exhibiting productive continuity between operations even if further details about the operations remain to be discovered). Sometimes further research reveals that what was taken to be a schema still contains gaps and must once again be treated as a sketch, albeit a more satisfactory one in which the earlier 
gaps have been filled (Bechtel 2009). Reasoning using sketches and schemas is a central part of reasoning in mechanistic sciences but requires that the scientist engage in mental activities (especially mental simulation) that are rather different from formal deductive inference.

Fourth, mechanistic explanations are inherently reductionistic insofar as they require specifying the parts of a mechanism and the operations the parts perform. But they also require consideration of the organization of the whole mechanism and its relation to conditions in its environment since it is only when appropriately situated that a mechanism will produce the phenomenon of interest. Mechanistic explanations are always multilevel accounts, integrating information about parts, operations, and organization within the mechanism with characterization of the phenomenon exhibited by the whole mechanism (Craver 2002, 2007; Craver and Bechtel 2007; Bechtel and Abrahamsen 2009).

Finally, although some philosophers of science have eschewed examining the process of scientific discovery, limiting their focus to justification, mechanism discovery is a rich topic for philosophical analysis. Bechtel and Richardson (1993/2010), while arguing that the heuristic of assuming that a mechanism is decomposable into operations localized in parts can be fallible, identified some of the experimental strategies used to pursue that heuristic. To discover the working parts of a mechanism, scientists may, for example, inhibit or stimulate a putative part and determine the effects on the overall phenomenon (e.g., add a suspected intermediate to a biochemical reaction and determine whether more of the end product is produced). Craver (2002) has also characterized techniques for intervening on the whole and detecting effects on parts (e.g., presenting a visual stimulus and recording changes in the firing rate of neurons in a particular region of the visual system). Darden and Craver (2002) described strategies for filling in sketches to produce schemas, and Darden (2006) has elaborated on strategies for revising accounts of mechanisms in the face of anomalies.

The new mechanistic philosophers of science have appealed to a variety of research endeavors in biology to ground their accounts of mechanism and mechanistic explanation. For example, Bechtel and Richardson focused on research into bioenergetics, biochemical genetics, and spatial memory, whereas Machamer, Darden, and Craver examined action potentials, long-term potentiation, and protein synthesis. Although there are biological phenomena to which these accounts may not apply (Skipper and Millstein [2005] question their applicability to natural selection), one of the major successes of the biological sciences in the nineteenth and twentieth centuries has been not just to identify numerous biological mechanisms but also to decompose them into their component parts and operations. Biologists have identified parts and operations involved in con- 
trol of the cell cycle, DNA repair and RNA editing, secretion and entry of molecules into cells and organelles, cell signaling, cell motility, and muscle contraction, to indicate just a few examples. Researchers focused on pathologies have identified genes and proteins involved in many illnesses, including diabetes, ulcers, and Williams syndrome.

Although in what follows I emphasize the limitations of this basic account of mechanisms, I readily acknowledge — based on these and many other exemplars - that it describes the conceptual framework in which the vast majority of productive research in biology has been conducted. I also embrace the features that differentiate mechanistic explanation from nomological explanation and the new perspectives on traditional philosophical issues it provides. My focus, however, is on a crucial limitation of the basic account that results from the emphasis on sequential organization so that a mechanism proceeds from start to finish conditions. At one point Machamer et al. acknowledge that mechanisms may employ "forks, joins, or cycles" $(2000,12)$, but they neither develop examples of such mechanisms nor explore the significance of such forms of organization for understanding how mechanisms behave. As I show below, nonsequential organization is not just a minor variant on sequential organization. When multiple parts of the mechanism can alter the execution of an operation associated with a specific part, researchers can no longer assume that the part will always perform the operation in the same way. How it does so may vary, depending on other operations, some seemingly downstream from it. As a result, researchers cannot understand the behavior of the whole mechanism by simply envisaging successive execution of operations but must seek other explanatory resources that allow them to factor in how other activity in the mechanism modulates specific operations.

Instead of emphasizing sequential execution of operations, Bechtel and Abrahamsen $(2005,423)$ speak of the "orchestrated functioning of the mechanism." Like a player in an orchestra, an individual part may behave differently as a result of operations performed by other parts. To illustrate how orchestrated functioning in biological mechanisms involves more than sequential operation, I will elaborate on cyclic organization of biological mechanisms that often takes the form of negative or positive feedback loops, which in turn make possible dynamic behaviors such as oscillations. ${ }^{5}$ Understanding such dynamics, as researchers who have em-

5. I am not claiming that such organization is found only in biological mechanisms. In designing mechanisms, engineers sometimes take inspiration from what is known about living organisms, and this could eventually lead to engineered systems that are constructed based wholly on the same design principles as biological mechanisms. Moreover, some natural phenomena, such as fires and storms, employ such organization. What I am claiming is that the modes of organization discussed here are crucial to living organisms and need to be considered in our accounts of them. 
braced systems approaches to biology increasingly have recognized, requires moving beyond qualitative characterizations and developing computational models. These enable researchers to characterize the various factors affecting each operation in the mechanism and account for how the mechanism as a whole changes its behavior through time. In the next section, I will show that such nonsequential organization is crucial to understanding how biological mechanisms support life.

3. Organisms as Autonomous Systems. Even while many biologists eagerly pursued basic mechanistic explanations in the nineteenth and twentieth centuries, vitalists focused on their limitations. The vitalists differed widely in their positive views about living phenomena but shared a common perception that mechanistic accounts were incapable of explaining the distinctive characteristics of living organisms. ${ }^{6}$ Particularly illuminating in this respect is Xavier Bichat, who embraced the mechanist project of decomposition as far as identifying 21 different types of tissue out of which he claimed the various organs of the human body were constituted. But he thought the properties of tissues were such that they could not be further explained by decomposing them into their parts and operations. He claimed, first, that the behavior of living organisms is too variable to be explained mechanically: "The instability of vital forces marks all vital phenomena with an irregularity which distinguishes them from physical phenomena [which are] remarkable for their uniformity" (Bichat 1805, 81). Moreover, this behavior involves distinctive forces that actively resist those forces operative in the inorganic world that would destroy the living tissue if left unopposed: "life is the sum of all those forces which resist death" (1).

While many mechanistically oriented biologists simply ignored the vitalists and pursued their research without questioning its assumptions, a few took the vitalists' objections seriously. Claude Bernard (1865) regarded Bichat's concerns about the irregularity of living systems as especially important since for him determinism was essential to scientific explanation. He countered Bichat by arguing that indeterminism was only apparent. By differentiating two environments - the internal environment in which the organs of an organism functioned and the external environment in which the organism as a whole functioned-he sought to demonstrate that strict determinism characterized operations when the appropriate environment was considered. Moreover, by claiming that internal operations are performed as needed to maintain the constancy of the internal

6. Many vitalists viewed themselves as natural scientists and construed their hypothesized vital forces as comparable to the forces Newton had identified as operative in inanimate matter. 
environment (even as forces in the external environment might cause them to change), he proposed a mechanistic account of how organisms resist death. A prominent example from his own research was the discovery that the liver converts glycogen to glucose whenever concentrations of glucose decline, thereby ensuring a constant supply for energy-demanding operations. Walter Cannon (1929) named this capacity homeostasis, and it came to be understood as involving negative feedback - a powerful way of organizing operations that subsequently was championed by the cyberneticists as a general control architecture for biological as well as social and engineered systems (Wiener 1948).

More than negative feedback is required to fully explain how organisms resist death, however. What is distinctive of living systems, beginning with single-celled organisms, is that they stand out against their environments as enduring, structured entities. Because they are subject to the normal physical processes that tend toward disorder (thermodynamic equilibrium or high entropy), they must perform the operations needed to maintain themselves in organized (nonequilibrium) states. Strictly, the second law of thermodynamics applies only to closed systems, whereas living systems are open systems that receive free energy from outside themselves. Although being an open system is necessary to maintain structure, it is not sufficient. In addition, energy (and matter) must be properly channeled so as to maintain the structure. This gives rise to an important feature of living systems - they are active systems regularly performing those operations that are needed to maintain themselves. Internally initiated activity is in fact one of the most noteworthy features of living systems, one recognized by Aristotle but too often neglected in the context of mechanistic research, in which the focus is on how the organism (or a mechanism isolated from it) responds to conditions set by the experimenter. But if one observes even single cells, they are endogenously active - changing shape and often moving and dividing. When not sleeping or hibernating, multicelled animals typically are moving in their environment. And even when sleeping or hibernating, they are still performing basic metabolic activities, including respiration, and their brains exhibit endogenously generated oscillations.

One reason such activity is required is that biological tissue, formed using relatively weak chemical bonds, is rather easily damaged by various physical forces. ${ }^{7}$ To maintain their structure, organisms must perform

7. See Collier and Hooker (1999, 244): "Living systems are not passively independent, in the way a rock's crystalline structure is undisturbable by all but the most violent signals from the environment. Rather, they are vulnerable to disruption by impinging signals - storms, predation, cold, . . .,- -and constantly in need of replenishing their dissipating energy and order. . . . Their structural bonds have energies measured in 

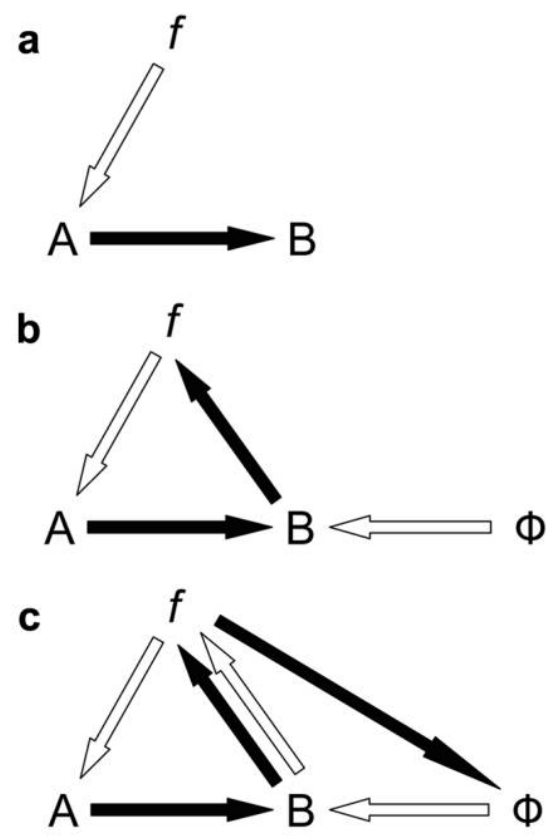

Figure 1. Representation of Rosen's (1991) proposal for a network of processes that execute self-repair. Open arrows represent the causal agent, while filled arrows represent the material transformed. Each node from which a filled arrow (a material change) originates has an open arrow (causal agency) coming into it from another node within the network.

repair operations. With humanly built machines, at least before our throwaway era, repair involved an external agent summoned to restore the machine to its proper operating condition, usually by replacing or rebuilding worn-down parts. For the most part, living organisms must manage repair by themselves (or die). Robert Rosen (1991) examined what is required for an organism to repair itself. Each repair function requires a causal metabolic process in which a substrate (A) is transformed into another (B), the part that is thereby repaired. This requires, as illustrated in figure $1 a$, a causal agent $(f)$, whose occurrence must be accounted for within the organism. Rosen allowed that $\mathrm{B}$ might provide the material from which $f$ might be constructed. Then, however, as indicated in figure $1 b$, another causal agent $(\Phi)$ must be provided to initiate the change of B into $f$. Now, the occurrence of this agent must be accounted for within

electron volts, even fractions thereof, not the millions of electron volts that fix a rock into responseless stability." 
the organism. While the material for making $\Phi$ might come from an existing component of the system (e.g., $f$ ), to avoid a regress of causal agents, something within the organism must initiate the change of $f$ into $\Phi$. By proposing, as shown in figure $1 c$, that $\mathrm{B}$ could perform this role, Rosen has arrived at a network in which all the causal processes responsible for repair originate within the mechanism. As evidenced in figure 1, a mechanism in which all repairs can be executed from within requires cyclic organization in which parts serve in the pathway leading to the occurrence of other parts that in turn figure in the pathway leading to their own repair.

In addition to possessing causal agents for all operations, an organism that can repair itself requires free energy. Accordingly, an organism must be positioned within a constant flow of free energy from a source to a sink, with the organism itself temporarily acting as the sink, trapping the energy and controlling its flow. Moreover, this energy is used by the organism to construct and repair itself and in particular to maintain the conditions on which its constitution and functioning depend. Ruiz-Mirazo, Peretó, and Moreno $(2004,330)$ apply the term autonomy to "a farfrom-equilibrium system that constitutes and maintains itself establishing an organizational identity of its own, a functionally integrated (homeostatic and active) unit based on a set of endergonic-exergonic couplings between internal self-constructing processes, as well as with other processes of interaction with its environment." This characterization identifies an autonomous system, not in terms of the particular material constituting it but rather in terms of its maintaining a continued existence as an organized system. ${ }^{8}$ Even an autonomous organism, though, can maintain itself only so long before it dies. What is relevant with respect to autonomy is not a permanent identity but continued existence for a relatively long period of time.

Such continued existence depends critically on coupling the energydemanding operations required to construct and maintain the organism with the free energy provided by virtue of being an open system. Collier and Hooker $(1999,243)$ emphasize how this requires integrated organization: "because their [autonomous systems'] capacities, i.e. their organised processes of interaction that ground their functional properties, must be so integrated that they are able to actively regenerate themselves, their

8. Some theorists do not consider a system to be autonomous unless it is able to adapt to varied circumstances and acts on its environment so as to ensure the conditions to maintain itself (Christiansen and Hooker 2000). It is useful, though, to first distinguish systems that internally manage the processes of generating and repairing themselves and then consider how further mechanisms could be added to them that enable adaptation to changing environmental circumstances. 
overall functionality can not be grounded in a mere aggregate of independent processes but requires that distinctive global process integration that alone insures regeneration of the whole as a joint interactive consequence across all their interrelated process cycles." Simple sequential organization allows only aggregation of component operations, and so it is insufficient to explain autonomy. Autonomous systems must employ a nonsequential or cyclic organization such as negative feedback so that when energy-demanding operations are called upon, the needed energyproviding operations are executed.

For researchers focused on decomposing biological systems to identify their component parts and operations, the coordination required to maintain autonomy is not a central concern. Rather, researchers concentrate on phenomena they elicit in experimental conditions in which they control the factors that may affect their preparation (e.g., a cell cultured in a specific medium). This research readily adopts the basic mechanistic framework, focusing on changes in the mechanism elicited experimentally. Only when researchers focus on recomposing the mechanism and resituating it in the organism (and the organism in its environment) might their attention be drawn to explaining features such as autonomy. Such a shift in focus is a major factor contributing to the recent interest in systems biology (Westerhoff and Palsson 2004; Noble 2006; Hofmeyr 2007).

4. Discovering and Theorizing about Cyclic Organization. While the previous section illustrates the importance of nonsequential organization if organisms are to be autonomous systems, more pragmatic considerations have often figured in the discovery of cyclic organization in living systems. For example, the first proposals of cycles in biochemistry resulted from practical necessity. The early physiological and biological chemists who set out to understand basic metabolic processes such as fermentation and respiration attempted to provide stepwise accounts of the phenomena they investigated, thus conforming to the basic account of mechanistic explanation. Knowing that oxidative metabolism converted glucose $\left(\mathrm{C}_{6} \mathrm{H}_{12} \mathrm{O}_{6}\right)$ into carbon dioxide and water and that this process would require multiple steps, these researchers searched for substances that could be possible intermediates. After identifying several 4-carbon compounds such as succinic $\left(\mathrm{C}_{4} \mathrm{H}_{6} \mathrm{O}_{4}\right)$ and fumaric $\left(\mathrm{C}_{4} \mathrm{H}_{4} \mathrm{O}_{4}\right)$ acids and 3-carbon compounds such as lactic $\left(\mathrm{C}_{3} \mathrm{H}_{6} \mathrm{O}_{3}\right)$ and pyruvic $\left(\mathrm{C}_{3} \mathrm{H}_{4} \mathrm{O}_{3}\right)$ acids, they proposed reactions by which each of these could be produced and further metabolized. Drawing on available evidence, Thunberg (1920) hypothesized a sequence of oxidation, hydration, and decarboxylation reactions to transform succinic acid into acetic acid: 


$$
\begin{gathered}
\text { succinic acid } \rightarrow \text { fumaric acid } \rightarrow \text { malic acid } \\
\rightarrow \text { oxaloacetic acid } \rightarrow \text { pyruvic acid } \rightarrow \text { acetic acid. }
\end{gathered}
$$

At this point, Thunberg confronted a problem: further removal of two hydrogen atoms from acetic acid $\left(\mathrm{C}_{2} \mathrm{H}_{4} \mathrm{O}_{2}\right)$ would not yield a known chemical compound. His solution was to propose that two molecules of acetic acid would combine; in the process, each would surrender a hydrogen atom, yielding succinic acid. Necessity thus led Thunberg to close the sequence of reactions for which he had direct evidence into a cycle (fig. $2 a$ ). He was prescient in proposing a cycle, but there were problems with the specifics of his account. Krebs and Johnson (1937) proposed an alternative arrangement of several of the key compounds in Thunberg's model in their citric acid cycle (later known as the Krebs' cycle). As shown in figure $2 b, \mathrm{Krebs}$ proposed that an unspecified 3-carbon compound derived from pyruvate (a product of glycolysis) entered the cycle by combining with oxaloacetic acid that had been generated in a previous iteration to produce citric acid and carbon dioxide. The citric acid was then metabolized through a sequence of reactions (primarily oxidations and decarboxylations), until oxaloacetic acid was formed once again.

Krebs might have been primed to find cyclic reactions in biochemical pathways as a result of his previous discovery of the ornithine cycle, in which urea is generated from the split of arginine into urea and ornithine and then reconstituted through the incorporation of ammonia $\left(\mathrm{NH}_{3}\right)$ groups (Krebs and Henseleit 1932). However, like Thunberg before him, he seems to have arrived at cyclic organization primarily because he could not piece together the known biochemical reactions in a sequential arrangement. After his discovery of this second cycle, though, Krebs (194648) was sufficiently intrigued to theorize about their significance. He characterized the ornithine and citric acid cycles as metabolic cycles and distinguished them from a type of cycle at a lower-level of organization that he termed enzyme cycles. Enzyme cycles, which he took to be comparable to catalytic processes in inanimate matter, such as the carbon-nitrogen cycle found in stars, involve metabolites that are reversibly converted between a reduced and an oxidized state. Metabolic cycles, he proposed, are cycles of enzyme cycles: "a metabolic cycle is a repetition of the pattern of the enzyme cycle on another plane of the chemical organization of living matter" (92). Figure 3 shows the citric acid cycle as a metabolic cycle consisting of enzyme cycles.

Claiming that "the metabolic cycle seems to have been specially evolved by living matter," Krebs probed its significance $(1946-48,96)$. As a first step, he noted that metabolic cycles can explain the apparent but not true reversibility of biochemical processes. The dissociation of water in hydrogen and oxygen ions is directly reversible, as is an enzyme cycle, but 


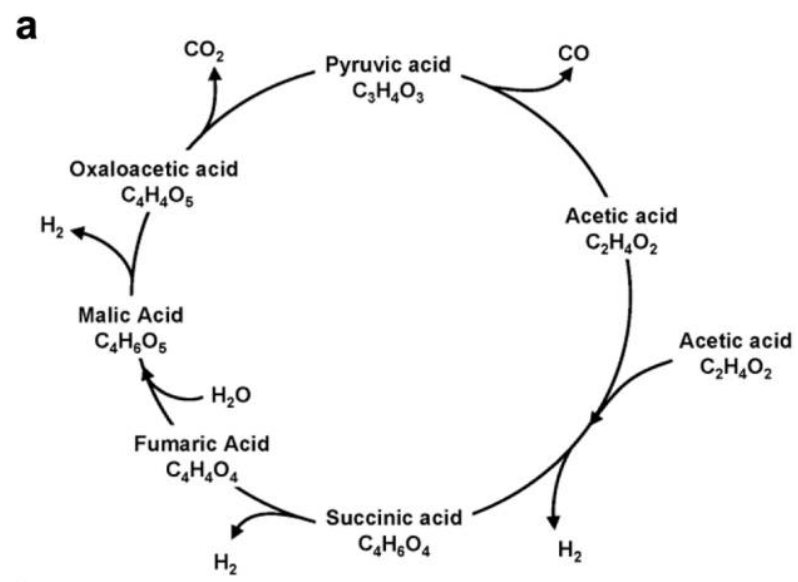

b

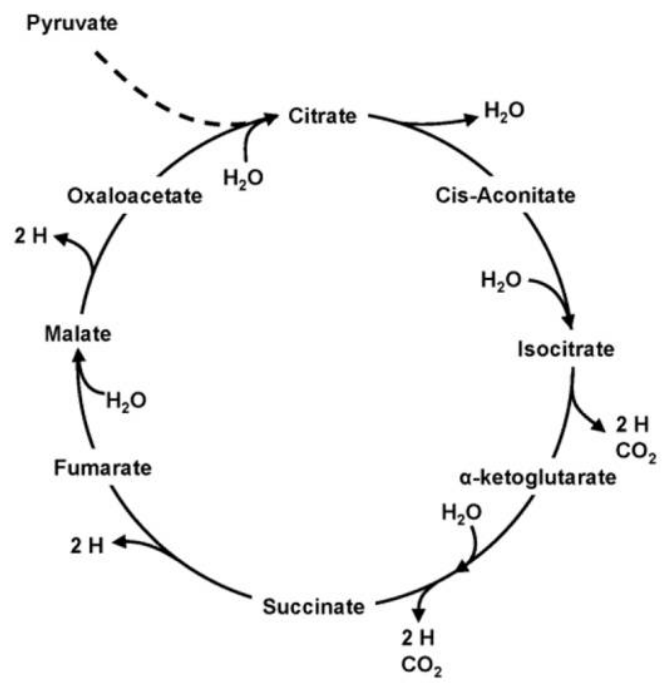

Figure 2. $a$, Cycle proposed by Thunberg (1920) to link various intermediates that figure in oxidative metabolism; $b$, citric acid cycle proposed by Krebs and Johnson (1937).

since the citric acid cycle involves different intermediates when proceeding from citrate to succinate than when going from succinate to citrate, it is not. The difference between reversible and cyclic reactions, Krebs goes on to suggest, contributes to explaining what Frederick Gowland Hopkins referred to as the dynamic equilibrium found in living systems. Unlike the equilibrium between dissociation of water into hydrogen and oxygen ions 


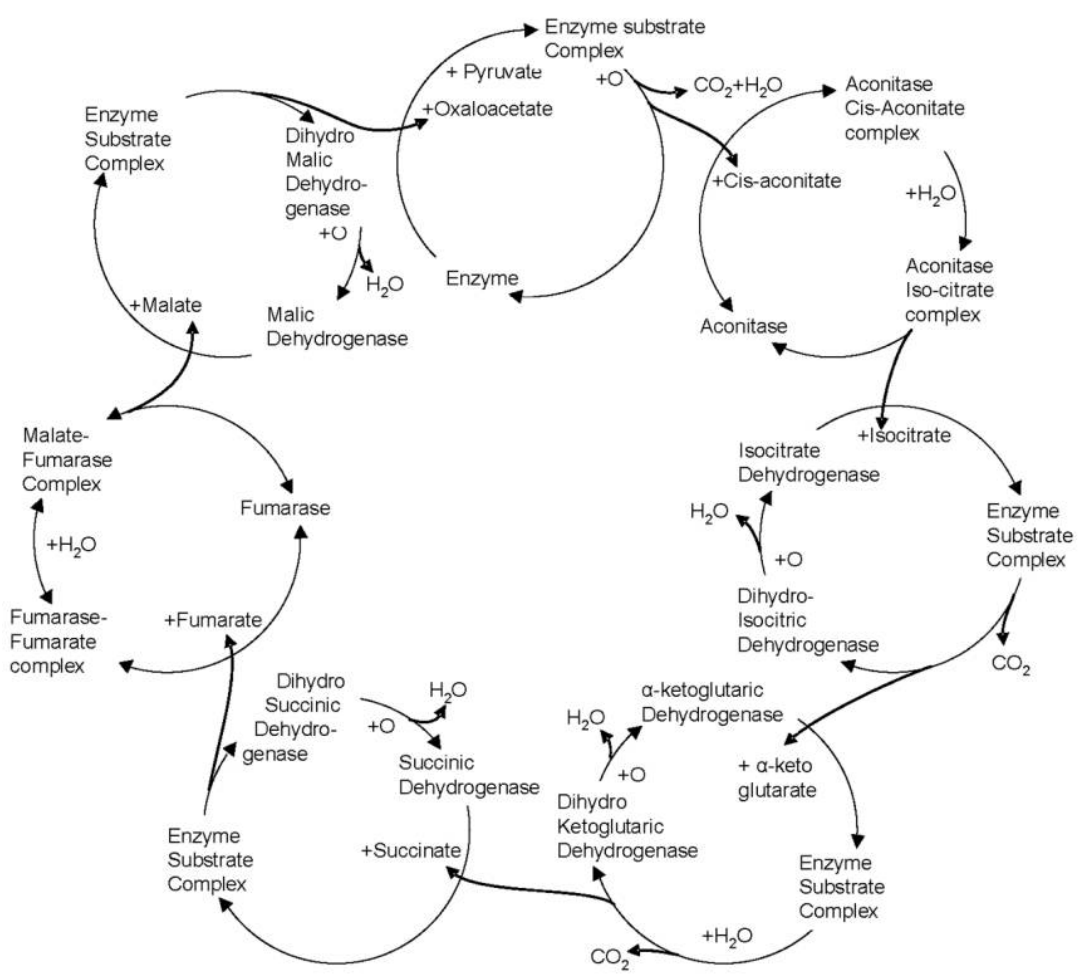

Figure 3. Representation of the citric acid cycle as a cycle of enzyme cycles (adapted from Krebs 1946-48).

and their reassociation into water molecules, a dynamic equilibrium requires work to rebuild a system that is constantly dissipating. Thus, Krebs argues, enzyme cycles and other reversible reactions are insufficient to maintain dynamic equilibrium: they "have little scope for producing work of any kind, be it mechanical work in muscle or chemical synthesis in glands or growing cells, or the osmotic work of excretion and absorption. Truly reversible reactions cannot result in any major changes within living matter; therefore they lead nowhere" (97). Metabolic cycles, in contrast, draw on an influx of new metabolites (carrying new free energy) to restore themselves to a condition in which further work can be performed. Thus, they provide a basis for explaining how living organisms can maintain themselves as enduring, active individuals. ${ }^{9}$ In these early speculations,

9. A related understanding of cyclic organization was advanced by the Hungarian chemist Tibor Gánti (2003), who sought to identify the simplest chemical system (what 
Krebs offers insights into how cyclic organization involves more than closed sequences of operations, in that they give rise to important characteristics of living systems such as their ability to capture free energy so as to execute subsequent work.

5. Endogenous Oscillatory Activity in Biological Mechanisms. In recruiting free energy to maintain itself, a cyclically organized mechanism satisfies a key requirement for maintaining endogenous activity, a feature that, I noted above, is characteristic of autonomous organisms. Biologists are increasingly recognizing signatures of this endogenous activity in the temporal dynamics of individual mechanisms. Endogenous activity is often manifested in periodic oscillations in which biological mechanisms cycle between phases driven by their own internal operations. Examples from three different types of biological mechanisms are as follows.

Ultradian physiological oscillations.-Many physiological oscillations have periods of well less than a day and are termed ultradian. One of the earliest periodic oscillations identified in biochemistry was glycolytic oscillation. While spectrographically measuring the concentration of NADH (reduced nicotinamide adenine dinucleotide) produced during glycolysis in yeast extract, Ghosh found that it oscillated with a period of about 1 minute (Chance, Estabrook, and Ghosh 1964). By 1979, a host of other oscillatory processes in cells with periods of less than 1 hour had been identified, including ones involving ion movements in mitochondria, cell membrane potentials (in neurons and other cells), muscle contractions, cell secretion, and cell movement (Rapp 1979). Subsequent research has further elaborated on these oscillations and their functions. For example, in brewer's yeast, Lloyd and Murray (2007) identified oscillations between oxidative and reductive phases over approximately 40 minutes, which they construed as a major regulator of cellular activity: processes such as cell division and gene expression are limited to the reductive phase, when DNA will not be damaged by free oxygen that would be available in the oxidative phase.

Oscillations in brain activity.-Berger's $(1929,1930)$ pioneering recordings of electrical activity at the scalp in an electroencephalogram (EEG) provided a window on the dynamics of the brain. The high temporal resolution reveals a complex signal, but Berger was able to identify oscillations with regular periodicity specific to a person's state: $(a)$ awake

he termed a chemoton) that would exhibit the features of a living system. He employed an abstract version of the citric acid cycle in the chemoton's metabolic mechanism, whose prime function was to make more of itself and generate a segregating membrane. For him as well as Krebs, cyclic organization was central to a mechanism remaking itself. For further discussion of Gánti, see Bechtel (2008, chap. 6) and Griesemer and Szathmáry (2008). 
with eyes closed yielded high-amplitude oscillations with a frequency (period) of $8-12 \mathrm{~Hz}$ that he called alpha rhythms, while (b) awake with eyes open and responding to stimuli yielded oscillations of lower amplitude and higher frequency (beta rhythms). He construed the alpha rhythms, produced without a stimulus, as reflecting endogenous activity. Subsequent research revealed that entering into sleep yields oscillations of lower frequency, while attention-demanding tasks generate high-frequency oscillations. EEG research continues today, with considerably improved spatial resolution, but newer technologies have provided different avenues for studying these oscillations. First, implanted electrodes make possible recordings of electrical activity in individual neurons. In invertebrates (Kandel 1976), and then in birds and mammals (Llinás 1988), conductances of sodium, potassium, and other ions were found to depend on continuously changing voltage over cell membranes. Llinás demonstrated that in many neurons these could give rise to spontaneous action potentials. A remarkable discovery of the past decade is that fMRI, which has been employed mainly to localize mental operations spatially, can be used to detect very low-frequency oscillations $(<.1 \mathrm{~Hz}$ ) that are synchronized across wide regions of human cortex (Fox and Raichle 2007). One network of synchronized oscillations is most active in what is called a resting state (in which participants are awake in a scanner but not required to perform a task), while other networks exhibit increased activity in task conditions. Mantini et al. (2007) identified six such anticorrelated networks; that is, regions within one network exhibit correlated oscillations with other regions in the same network but uncorrelated oscillations with regions in the five other networks. Moreover, they found that the higher-frequency oscillations found with EEG were temporally embedded within these low-frequency oscillations found with fMRI. Findings such as these have led a number of neuroscientists to adopt a view of the brain as endogenously active. Exogenous stimuli may perturb activity in the brain, but they do not initiate it (Buzsáki 2006).

Circadian rhythms. - Conventionally, oscillations with periods of approximately 24 hours are referred to as circadian, so as to distinguish them from ultradian rhythms. Circadian rhythms regulate a vast range of physiological and behavioral functions (as reflected in such measures as heart rate, metabolic rate, and reaction time) in living organisms from bacteria to plants and animals (Dunlap, Loros, and DeCoursey 2004). Maintenance of such rhythms enables organisms to coordinate their internal and external activities with the 24-hour day/night cycle on our planet. The basic mechanisms responsible for their maintenance are intracellular, as discussed below.

6. Cyclic Organization and Endogenously Active Mechanisms. Many mechanisms responsible for endogenous oscillations have been identified, and, 
not surprisingly, they have been found to make use of cyclic organization involving both negative and positive feedback loops. ${ }^{10}$ In glycolytic oscillations, for example, the crucial feedback loops converge in phosphofructokinase, an allosteric enzyme excited by its own products (adenosine diphosphate and fructose diphosphate) and inhibited by one of its inputs (adenosine triphosphate [ATP], which in turn is generated in yet larger quantities several steps later in the pathway). Circadian oscillations provide another clear example: except in cyanobacteria, the responsible mechanism employs feedback loops in which the transcription and translation of a gene produces a protein that, after transport back into the nucleus, inhibits its own transcription until it is subsequently broken down. This negative feedback loop is coupled with a positive feedback loop in which another gene product excites its own transcription and translation. Cycles and feedback make possible, but do not guarantee, ongoing oscillatory activity. In addition, these mechanisms are thermodynamically open and involve reactions whose mathematical representation is nonlinear (for additional details, see Bechtel and Abrahamsen [2011]).

Oscillations are symptoms of endogenous activity, but they are also an important factor in maintaining endogenous activity. They are especially important for ensuring that organisms regularly perform the various operations that are required to maintain themselves. In particular, infradian oscillations ensure that organisms repeatedly execute operations required to procure energy and to synthesize new proteins, despite the fact that these operations are incompatible with each other. Circadian oscillations coordinate behavior over the course of the day so that, for example, animals forage for food and sleep, each at times that minimize risks such as predation.

Despite the importance of endogenous oscillations, researchers studying biological mechanisms often treat the resulting variability simply as noise in their data to be filtered out by averaging measurements over time and individuals. While this practice yields cleaner data sets for purposes of description or detecting responses to interventions, it also masks the effects of endogenous oscillations that may be important for the very phenomenon being investigated. To cite just one example, the endogenous activity in brains (noted above) may be responsible for variations in behavioral responses to sensory stimuli (Fox et al. 2007). The resulting behavior is a product of both the endogenous activity and the stimulus. Moreover, endogenous brain activity may be critical for coordinating different op-

10. Engineers have long known that feedback loops can generate oscillations, but typically their objective is to dampen them. For example, a heating system with a thermostat produces temperature oscillations, which the heating and cooling industry has tried to minimize so as to keep temperatures very close to the target. 
erations required in a given task since relatively weak signals can generate synchronous oscillations and, hence, coordinated responses from diverse brain regions, with little energy expenditure (Buzsáki 2006).

7. From Basic to Dynamic Mechanistic Explanations. In the preceding sections, I have emphasized the crucial role of cyclic organization and the endogenous activity it supports in enabling biological organisms to maintain autonomy. The basic mechanistic account, as articulated by Machamer et al. (2000), by treating mechanisms as operating sequentially from start to finish conditions, is insufficient to accommodate such cyclic organization and the endogenous dynamics to which mechanisms give rise and, hence, how they enable organisms to maintain autonomy. Even though the authors acknowledge the possibility of cycles, they treat them as just a variant on sequential organization and do not consider the consequences for the behavior of mechanisms. Cycles are not just a variant on sequential organization, and they result in very different behavior. With nonsequential organization, the operations performed by parts of the mechanism vary dynamically, depending on activity elsewhere in the mechanism, undermining the ability to understand how the mechanisms will behave simply by mentally stepping through the operations from start to termination conditions. Patterns such as oscillations become crucial for understanding a mechanism's behavior.

I noted earlier that the sequential perspective is not an arbitrary imposition in basic mechanistic accounts. Biologists commonly represent to themselves the activity of a biological mechanism by mentally rehearsing its operations in sequence. For example, in the case of glycolysis, biologists commonly characterize the sequence of reactions as starting with glucose and ending with alcohol or lactic acid. The feedback loops, whereby ATP generated in later steps of glycolysis both is consumed in early steps and regulates the behavior of an allosteric enzyme (phosphofructokinase), are noted, but their potential for generating glycolytic oscillations is seldom recognized. When nonsequential organization, such as negative feedback, is included in accounts of a mechanism's organization, it is conceived as comparable to a thermostat in keeping the mechanism within target operating conditions. The oscillations that result are treated as noise and ignored.

One reason biologists often limit themselves to basic mechanisms in explaining biological phenomena is that their primary tools are those for decomposing mechanisms into parts and operations. When they try to recompose them, they do so qualitatively, rehearsing in sequence the different operations in their minds. However, when operations conceived as occurring later alter operations conceived as earlier and the operations change continuously through time, it is no longer feasible to keep track 


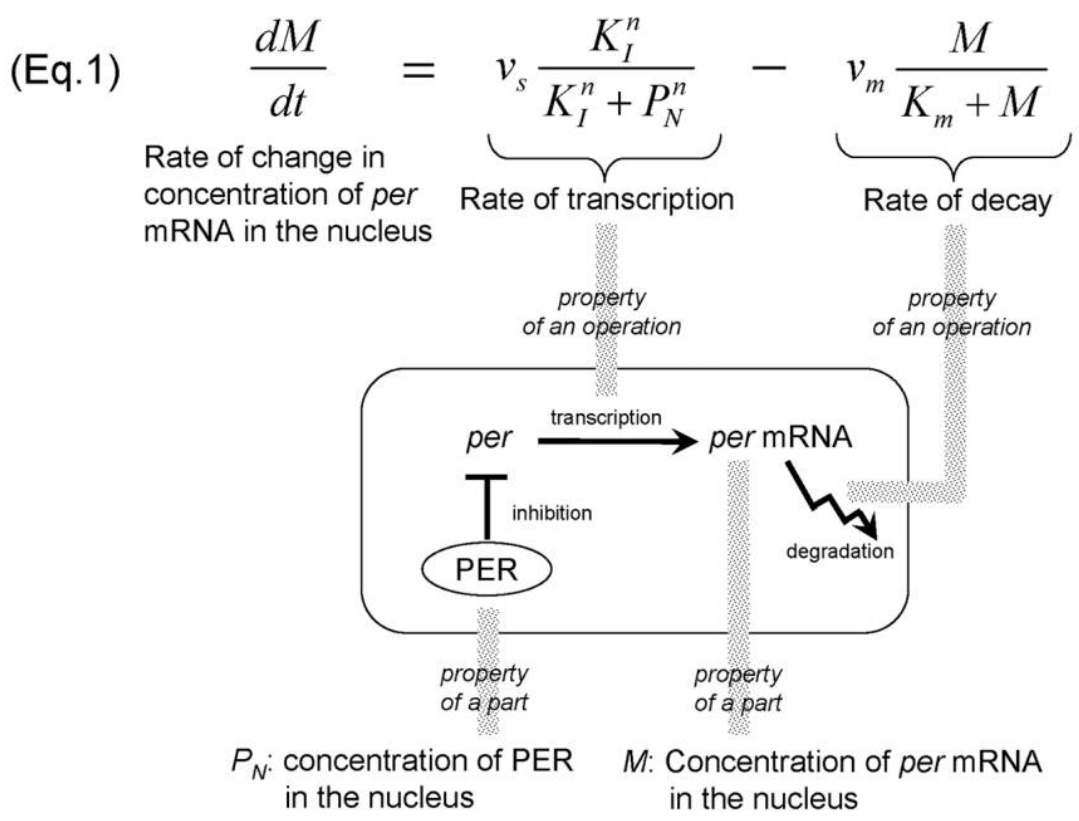

Figure 4. Representation of the project of describing the orchestrated functioning of a mechanism in equations. Operations in the mechanism for generating circadian rhythms in Drosophila - the transcription of per into per mRNA and the degradation of per mRNA - are represented in the first equation in Goldbeter's (1995) model.

of the various conditions that at a given moment affect the operation of a specific part. The additional tools needed to understand such systems are computational modeling and dynamic systems analysis. Computational modeling of a mechanism captures its temporal dynamics in equations using variables grounded in a mechanistic account. Usually these are systems of differential equations in which the variables and other terms correspond to properties of the mechanism's parts and operations. Tools of dynamic systems theory, such as representing the trajectory of a system through a phase space in which attractors are identified, serve to both render the behavior of the mechanism more intelligible and guide further development of the model.

An example illustrates the value of computational modeling for understanding a mechanism: to determine whether a mechanism in which a protein inhibits its own transcription could generate sustained circadian oscillations, Goldbeter (1995) represented the proposed mechanism in five differential equations. Figure 4 shows how the first equation in his model 
relates changes in the concentration of per mRNA $(M)$ to two operations in the feedback mechanism proposed by Hardin, Hall, and Rosbash (1990) for Drosophila: one in which the transcription of the gene per is modulated by the concentration of the protein PER in the nucleus $\left(P_{N}\right)$ and a second in which per mRNA degrades in cytoplasm. The rate of each operation corresponds to one of the terms in this equation. Goldbeter then ran simulations by supplying both initial values for variables such as $M$ and $P_{N}$ and best-fit or biologically plausible values for parameters such as $v_{s}$, $v_{m}$, and $n$ and iteratively applying these equations. These simulations showed that by using parameter values that Goldbeter maintained were biologically plausible, the values of the variables $M$ and $P_{N}$ oscillate, without dampening, at a constant phase offset. Moreover, this relation is restored if perturbed (in dynamic systems terms, the values produced a limit cycle). He thereby demonstrated that the proposed mechanism was capable of accounting for circadian rhythms. ${ }^{11}$

In this simple illustration, the computational model and its analysis in terms of a cyclic attractor simply confirmed that the mechanism could generate endogenous oscillations. However, the framework is readily generalized to accommodate multiple interactions between components of the mechanism. Modelers can add terms to characterize how conditions elsewhere in the mechanism modulate specific parts of the mechanism and include complex nonlinear relations between components. Such computational modeling is essential to understanding the behavior of mechanisms involving nonsequential arrangement of nonlinear operations.

Reliance on simulations that use equations to understand the behavior of mechanisms may appear to depart from the mechanistic perspective and embrace something very much like the DN account of explanation. A simulation involves deriving values for variables at subsequent times from the equations and values at an initial time. However, simulations are crucially different from DN explanations. First, the equations are advanced not as general laws but as descriptions of the operations of specific parts of a mechanism. Second, the purpose of a computational simulation (like mental simulation in the basic mechanistic account) is not to derive the phenomenon being explained but to determine whether the proposed mechanism would exhibit the phenomenon. Finally, an important part of evaluating the adequacy of a computational model is that the parts and operations it describes are those that can be discovered through traditional techniques for decomposing mechanisms. The need

11. Shortly afterward, many additional components of the circadian mechanism in Drosophila and other species were identified (see Bell-Pedersen et al. 2005, for a review). Leloup and Goldbeter (2008) as well as others have developed computational models of these proposed mechanisms. 
for simulation with mathematical models shows how adequately recomposing the sorts of mechanisms commonly encountered in biology and understanding their dynamics requires tools beyond the qualitative one contemplated in the basic mechanistic account. Accordingly, Bechtel and Abrahamsen (2010) characterize such explanations as dynamic mechanistic explanations.

8. Conclusion. Ever since the vitalists challenged the mechanisms proposed by eighteenth- and nineteenth-century biologists, mechanistic explanations have been criticized regularly for their inability to account for important biological phenomena. Repeatedly, though, biologists have expanded their understanding of mechanisms to handle the recalcitrant phenomena. The recognition of the importance of cyclic organization and the resulting temporal dynamics in biological mechanisms so as to explain the autonomy of living systems are further instances of this expansion. While it might seem unfair that mechanists revise their conception of a mechanism in the face of an apparently recalcitrant phenomenon, such a process of revision constitutes a productive discovery process. Indeed, since the critics often lack a positive research program of their own, their main contribution can be viewed as prodding the development of more adequate conceptions of mechanisms. My contention has been that the basic account of mechanistic explanation likewise must be extended to deal with biological mechanisms whose operations are not sequential but involve cyclic organization through which operations elsewhere in the mechanism alter the execution of individual operations. Mentally rehearsing operations sequentially is not sufficient to determine how such a mechanism will behave, and the basic mechanistic account must be extended in the direction of dynamic mechanistic explanation in which computational modeling and dynamic systems analysis is invoked to understand the dynamic behavior of biological mechanisms.

\section{REFERENCES}

Bechtel, William. 2008. Mental Mechanisms. London: Routledge.

2009. "Generalization and Discovery by Assuming Conserved Mechanisms: CrossSpecies Research on Circadian Oscillators." Philosophy of Science 76:762-73.

Bechtel, William, and Adele Abrahamsen. 2005. "Explanation: A Mechanist Alternative." Studies in History and Philosophy of Biological and Biomedical Sciences 36:421-41.

- 2009. "Decomposing, Recomposing, and Situating Circadian Mechanisms: Three Tasks in Developing Mechanistic Explanations." In Reduction and Elimination in Philosophy of Mind and Philosophy of Neuroscience, ed. H. Leitgeb and A. Hieke, 173-86. Frankfurt: Ontos.

$\rightarrow$ - 2010. "Dynamic Mechanistic Explanation: Computational Modeling of Circadian Rhythms as an Exemplar for Cognitive Science." Studies in History and Philosophy of Science A 41:321-33.

—. 2011. "Complex Biological Mechanisms: Cyclic, Oscillatory, and Autonomous." In 
Philosophy of Complex Systems: Handbook of the Philosophy of Science, vol. 10, ed. Clifford A. Hooker, 257-85. New York: Elsevier.

Bechtel, William, and Robert C. Richardson. 1993/2010. Discovering Complexity: Decomposition and Localization as Strategies in Scientific Research. Repr., Cambridge, MA: MIT Press.

Bell-Pedersen, Deborah, Vincent M. Cassone, David J. Earnest, Susan S. Golden, Paul E. Hardin, Terry L. Thomas, and Mark J. Zoran. 2005. "Circadian Rhythms from Multiple Oscillators: Lessons from Diverse Organisms." Nature Reviews Genetics 6:544-56.

Berger, Hans. 1929. "Über Daas Elektroenkephalogramm Des Menschen." Archiv für Psychiatrie und Nervenkrankheiten 87:527-70.

_. 1930. "Über Daas Elektroenkephalogramm Des Menschen: Zweite Mitteilung." Journal für Psychologie und Neurologie 40:160-79.

Bernard, Claude. 1865. An Introduction to the Study of Experimental Medicine. New York: Dover.

Bichat, Xavier. 1805. Recherches physiologiques sur la vie et la mort. 3rd ed. Paris: Machant.

$\rightarrow$ Boas, Marie. 1952. "The Establishment of the Mechanical Philosophy." Osiris 10:412-541.

Buzsáki, György. 2006. Rhythms of the Brain. Oxford: Oxford University Press.

Cannon, Walter B. 1929. "Organization of Physiological Homeostasis." Physiological Reviews 9:399-431.

Chance, Britton, Ronald W. Estabrook, and A. Ghosh. 1964. "Damped Sinusoidal Oscillations of Cytoplasmic Reduced Pyridine Nucleotide in Yeast Cells." Proceedings of the National Academy of Sciences 51:1244-51.

Christiansen, Wayne D., and Clifford A. Hooker. 2000. "Autonomy and the Emergence of Intelligence: Organised Interactive Construction." Communication and Cognition: Artificial Intelligence 17:133-57.

Collier, John D., and Clifford A. Hooker. 1999. "Complexly Organised Dynamical Systems." Open Systems and Information Dynamics 6:241-302.

Craver, Carl F. 2002. "Interlevel Experiments and Multilevel Mechanisms in the Neuroscience of Memory." Philosophy of Science 69 (Proceedings): S83-S97.

. 2007. Explaining the Brain: What a Science of the Mind-Brain Could Be. New York: Oxford University Press.

$\rightarrow$ Craver, Carl F., and William Bechtel. 2007. "Top-Down Causation without Top-Down Causes." Biology and Philosophy 22:547-63.

Darden, Lindley. 2006. Reasoning in Biological Discoveries: Essays on Mechanisms, Interfield Relations, and Anomaly Resolution. Cambridge: Cambridge University Press.

$\rightarrow$ Darden, Lindley, and Carl Craver. 2002. "Strategies in the Interfield Discovery of the Mechanism of Protein Synthesis." Studies in History and Philosophy of Biological and Biomedical Sciences 33:1-28.

Dunlap, Jay C., Jennifer J. Loros, and Patricia J. DeCoursey, eds. 2004. Chronobiology: Biological Timekeeping. Sunderland, MA: Sinauer.

Fox, Michael D., and Marcus E. Raichle. 2007. "Spontaneous Fluctuations in Brain Activity Observed with Functional Magnetic Resonance Imaging." Nature Reviews Neuroscience 8:700-711.

Fox, Michael D., Abraham Z. Snyder, Justin L. Vincent, and Marcus E. Raichle. 2007. "Intrinsic Fluctuations within Cortical Systems Account for Intertrial Variability in Human Behavior." Neuron 56:171-84.

Gánti, Tibor. 2003. The Principles of Life. New York: Oxford.

$\rightarrow$ Glennan, Stuart. 1996. "Mechanisms and the Nature of Causation." Erkenntnis 44:50-71.

- 2002. "Rethinking Mechanistic Explanation." Philosophy of Science 69 (Proceedings): S342-S353.

Goldbeter, Albert. 1995. "A Model for Circadian Oscillations in the Drosophila Period Protein (Per)." Proceedings of the Royal Society of London B 261:319-24.

Griesemer, James R., and Eörs Szathmáry. 2008. "Gánti’s Chemoton Model and Life Criteria." In Protocells: Bridging Nonliving and Living Matter, ed. Steen Rasmussen, Liaohai Chen, Norman Packard, Mark Bedau, David Deamer, Peter Stadler, and David Krakauer. Cambridge, MA: MIT Press.

Hardin, Paul E., Jeffrey C. Hall, and Michael Rosbash. 1990. "Feedback of the Drosophila 
Period Gene Product on Circadian Cycling of Its Messenger RNA Levels." Nature 343: $536-40$.

Hempel, Carl G. 1965. "Aspects of Scientific Explanation.” In Aspects of Scientific Explanation and Other Essays in the Philosophy of Science, ed. Carl G. Hempel, 331-496. New York: Macmillan.

Hofmeyr, Jan-Hendrik S. 2007. "The Biochemical Factory That Autonomously Fabricates Itself: A Systems Biological View of the Living Cell.” In Systems Biology, ed. Fred C. Boogerd, Frank J. Bruggeman, Jan-Hendrik S. Hofmeyr, and Hans V. Westerhoff, 21742. Amsterdam: Elsevier.

Kandel, Eric R. 1976. Cellular Basis of Behavior: An Introduction to Behavioral Neurobiology. San Francisco: W. H. Freeman.

Kauffman, Stuart. 1971. "Articulation of Parts Explanations in Biology and the Rational Search for Them.” In PSA, 1970, ed. Robert C. Bluck and Robert S. Cohen, 257-72. Dordrecht: Reidel.

Kosslyn, Stephen Michael. 1994. Image and Brain: The Resolution of the Imagery Debate. Cambridge, MA: MIT Press.

Krebs, Hans Adolf. 1946-48. "Cyclic Processes in Living Matter." Enzymologia 12:88-100.

Krebs, Hans Adolf, and Kurt Henseleit. 1932. "Untersuchungen Über Die Harnstoffbildung Im Tierkörper." Zeitschrift für physiologische Chemie 210:33-46.

Krebs, Hans Adolf, and William Arthur Johnson. 1937. "The Role of Citric Acid in Intermediate Metabolism in Animal Tissues." Enzymologia 4:148-56.

Leloup, Jean-Christophe, and Albert Goldbeter. 2008. "Modeling the Circadian Clock: From Molecular Mechanism to Physiological Disorders." BioEssays 30:590-600.

Llinás, Rodolfo R. 1988. "The Intrinsic Electrophysiological Properties of Mammalian Neurons: Insights into Central Nervous System Function." Science 242:1654-64.

Lloyd, David, and Douglas B. Murray. 2007. "Redox Rhythmicity: Clocks at the Core of Temporal Coherence." BioEssays 29:465-73.

Machamer, Peter, Lindley Darden, and Carl F. Craver. 2000. "Thinking about Mechanisms." Philosophy of Science 67:1-25.

Mantini, D., M. G. Perrucci, C. Del Gratta, G. L. Romani, and Maurizio Corbetta. 2007. "Electrophysiological Signatures of Resting State Networks in the Human Brain." Proceedings of the National Academy of Sciences 104:13170-75.

Noble, Denis. 2006. The Music of Life: Biology beyond the Genome. Oxford: Oxford University Press.

Rapp, P. E. 1979. “An Atlas of Cellular Oscillators.” Journal of Experimental Biology 81: 281-306.

$\rightarrow$ Riskin, Jessica. 2003. "The Defecating Duck; or, The Ambiguous Origins of Artificial Life." Critical Inquiry 29:599-633.

Rosen, Robert. 1991. Life Itself: A Comprehensive Inquiry into the Nature, Origin, and Fabrication of Life. New York: Columbia University Press.

Ruiz-Mirazo, Kepa, Juli Peretó, and Alvaro Moreno. 2004. "A Universal Definition of Life: Autonomy and Open-Ended Evolution." Origins of Life and Evolution of the Biosphere 34:323-46.

Simon, Herbert A. 1962. "The Architecture of Complexity: Hierarchic Systems." Proceedings of the American Philosophical Society 106:467-82.

Skipper, Robert A., and Roberta L. Millstein. 2005. "Thinking about Evolutionary Mechanisms: Natural Selection." Studies in History and Philosophy of Biological and Biomedical Sciences 36:327-47.

Thagard, Paul. 2003. "Pathways to Biomedical Discovery." Philosophy of Science 70:23554.

. 2006. Hot Thought: Mechanisms and Applications of Emotional Cognition. Cambridge, MA: MIT Press.

Thunberg, Torsten Ludvig. 1920. "Zur Kenntnis Des Intermediären Stoffwechsels Und Der Dabei Wirksamen Enzyme.” Skandinavisches Archiv für Physiologie 40:9-91.

$\rightarrow$ Westerhoff, Hans V., and Bernhard O. Palsson. 2004. "The Evolution of Molecular Biology into Systems Biology.” Nat Biotech 22:1249-52. 
Wiener, Norbert. 1948. Cybernetics; or, Control and Communication in the Animal and the Machine. New York: Wiley.

Wimsatt, William C. 1976. "Reductionism, Levels of Organization, and the Mind-Body Problem." In Consciousness and the Brain: A Scientific and Philosophical Inquiry, ed. G. Globus, G. Maxwell, and I. Savodnik, 202-67. New York: Plenum. 